\title{
BIBECHANA
}

A Multidisciplinary Journal of Science, Technology and Mathematics

ISSN 2091-0762 (Print), 2382-5340 (Online)

Journal homepage: http://nepjol.info/index.php/BIBECHANA

Publisher: Research Council of Science and Technology, Biratnagar, Nepal

\section{Analytical solution of system of differential equations by variational iteration method}

\author{
Jamshad Ahmed $^{1 *}$, Faizan Hussain ${ }^{2,3}$ \\ ${ }^{1}$ Department of Mathematics, Faculty of Sciences, University of Gujrat, Pakistan \\ ${ }^{2}$ Department of Mathematics, University of Gujrat, (Sialkot Campus) Pakistan \\ ${ }^{3}$ Department of Mathematics, NCBA\&E, Gujrat (Sub-Campus) Pakistan \\ "Email: jamshadahmadm@gmail.com
}

Article history: Received 11 March, 2015; Accepted 17 September, 2015

DOI: http://dx.doi.org/10.3126/bibechana.v13i0.13394

\section{Abstract}

In this paper, Varitational Iteration Method using He's Polynomials is used to construct the exact as well as approximate solutions of differential equations. From the obtained numerical results, it has been observed that this proposed technique is very efficient and reliable for the solution of the linear and nonlinear system of differential equations. Numerical results and graphical representation reflect the accuracy and effectiveness of the proposed modification.

ORCOST: All rights reserved.

Keywords: Variational interation method; He's Polynomials; System of linear and non-linear differential equations.

\section{Introduction}

In recent years considerable interest in system of ordinary differential equations has been stimulated due to their numerous applications in the fields of physics and engineering. A huge number of research and investigations have been invested in these directions. In this paper we consider a very efficient and power full technique He's variational iteration method for finding approximate solutions of systems of differential equations. This technique was first time introduce by the Chinese mathematician He [1]. The variational interaion method is used to investigate autonomous ordinary differential systems [2], Helmholtz equation [3], Burger's and coupled Burger's equation [4], Coupled Schrodinger-KdV equations and shallow water equations [4]. Also the application of the present technique to linear fractional partial differential equations arising from fluid mechanics is presented in [5]. The variational iteration method is investigated in [6-10] to solve parabolic integro-differential equations. This method is 
applied to find the solution of various classes of variational problem [11]. Some recent research works in this field are [12-20].

\section{Analysis of VIM for System of Differential Equations}

In case of $m$ equations, we rewrite equations in the form

$$
L_{i}\left(y_{i}\right)+N_{i}\left(y_{1}+y_{2}+\cdots+y_{m}\right)=f_{i}(x), i=1,2, \cdots m,
$$

where $L_{i}$ is a linear with respect to $y_{i}$ and $N_{i}$ is the nonlinear part of $i$ th equation. In this case the correct functional are obtained as

$$
y_{i, n+1}(x)=y_{i, n}(x)+\int_{0}^{x} \lambda_{i}(\tau, x)\left(L_{i}\left(y_{i}(\tau)\right)+N_{i}\left(\tilde{y}_{1, n}(\tau), \tilde{y}_{2, n}(\tau), \cdots, \tilde{y}_{m, n}(\tau)\right)-f(\tau)\right) d \tau,
$$

and the optimal values of $\lambda_{i}, i=1,2, \cdots, m$, are obtained by taking the variation from both sides of the functional and finding stationary condition using

$$
\delta y_{i, n+1}=0, i=1,2, \cdots, m \text {. }
$$

Our goal in the paper is the use of the following system of sequences instead of the system which results from the Variational iteration method:

$$
y_{i, n+1}(x)=y_{i, n}(x)+\int_{0}^{x} \lambda_{i}(\tau, x)\left(L_{i}\left(y_{i}(\tau)\right)+N_{i}\left(y_{1, n}(\tau), y_{2, n}(\tau), \cdots, y_{m, n}(\tau)\right)-f(\tau)\right) d \tau,
$$

For $i=2, \cdots, m$. In fact the updated values $y_{1, n+1}, y_{2, n+2}, \ldots, y_{(i-1),(n+1)}$, are used for finding $y_{i,(n+1)}$. This technique accelerates the convergence of the system of sequences. Therefore, using just few terms of the sequences, an accurate solution can be obtained for a large domain of the problem.

\section{Analysis of VIM using He's Polynomials}

Variational Iteration Method using He's polynomials [21] is a modified form of VIM. This modification is obtained by coupling the correction functional of VIM with He's polynomials and is given by

$$
\sum_{n=0}^{\infty} p^{(n)} y_{i, n+1}(x)=y_{i, 0}(x)+p \int_{0}^{x} \lambda_{i}(\tau, x)\left(\sum_{n=0}^{\infty} p^{(n)} L_{i}\left(y_{i, n}\right)+\sum_{n=0}^{\infty} p^{(n)} N_{i, n}\left(\tilde{y}_{i, n}\right)-f_{i}(\tau)\right) d \tau, i=1,2, \ldots, m
$$

By comparing the like powers of $p$, give solution of various order.

\section{Numerical Applications}

Example 4.1 Consider the system of first order differential equations,

$$
\begin{aligned}
& y_{1}^{\prime}=y_{3}-\cos x, \\
& y_{2}^{\prime}=y_{3}-e^{x},
\end{aligned}
$$




$$
y_{3}^{\prime}=y_{1}-y_{2}
$$

subjected to the initial conditions

$$
y_{1}(0)=1, y_{2}(0)=0, y_{3}(0)=2 \text {. }
$$

According to VIM, correction functional for Eq. (4), (5) \& (6) can be written as,

$$
\left\{\begin{array}{l}
y_{1, n+1}(x)=y_{1, n}(x)+\int_{0}^{x} \lambda_{1}(x, \tau)\left(\frac{d y_{1, n}(\tau)}{d \tau}-\tilde{y}_{3, n}+\cos \tau\right) d \tau, \\
y_{2, n+1}(x)=y_{2, n}(x)+\int_{0}^{x} \lambda_{2}(x, \tau)\left(\frac{d y_{2, n}}{d \tau}-\tilde{y}_{3, n}+\tilde{e}^{\tau}\right) d \tau, \\
y_{3, n+1}(x)=y_{3, n}(x)+\int_{0}^{x} \lambda_{3}(x, \tau)\left(\frac{d y_{3, n}}{d \tau}-\tilde{y}_{1, n}+\tilde{y}_{2, n}\right) d \tau,
\end{array}\right.
$$

The Lagrange multiplier $\lambda_{i}(x, \tau), i=1,2,3$ can be identified via variational theory; i.e. the multiplier should be chosen in such a way that the correction functional equation is stationary i.e. $\delta y_{1, n+1}(x)=0, \delta y_{2, n+1}(x)=0 \& \delta y_{3, n+1}(x)=0$. From Eq. (7)

$$
\lambda_{1}(x, \tau)=-1, \lambda_{2}(x, \tau)=-1, \text { and } \lambda_{3}(x, \tau)=-1,
$$

Thus Eq. (7) becomes

$$
\left\{\begin{array}{l}
y_{1, n+1}(x)=y_{1, n}(x)+\int_{0}^{x}(-1)\left(\frac{d y_{1, n}(\tau)}{d \tau}-y_{3, n}+\cos \tau\right) d \tau \\
y_{2, n+1}(x)=y_{2, n}(x)+\int_{0}^{x}(-1)\left(\frac{d y_{2, n}}{d \tau}-y_{3, n}+e^{\tau}\right) d \tau \\
y_{3, n+1}(x)=y_{3, n}(x)+\int_{0}^{x}(-1)\left(\frac{d y_{3, n}}{d \tau}-y_{1, n}+y_{2, n}\right) d \tau
\end{array}\right.
$$

for $n=0$, Eq. (8) gives,

$$
\left\{\begin{array}{l}
y_{1,0}(x)=1-\sin x, \\
y_{2,0}(x)=1-e^{x}, \\
y_{3,0}(x)=2,
\end{array}\right.
$$

for $n=1$, Eq. (8) gives, 


$$
\begin{aligned}
& \left\{\begin{array}{l}
y_{1,1}(x)=y_{1,0}(x)+\int_{0}^{x}(-1)\left(\frac{d y_{1,0}(\tau)}{d \tau}-y_{3,0}+\cos \tau\right) d \tau, \\
y_{2,1}(x)=y_{2,0}(x)+\int_{0}^{x}(-1)\left(\frac{d y_{2,0}}{d \tau}-y_{3,0}+e^{\tau}\right) d \tau, \\
y_{3,1}(x)=y_{3,0}(x)+\int_{0}^{x}(-1)\left(\frac{d y_{3,0}}{d \tau}-y_{1,0}+y_{2,0}\right) d \tau,
\end{array}\right. \\
& \left\{\begin{array}{l}
y_{1,2}(x)=e^{x} \\
y_{2,2}(x)=\sin x \\
y_{3,2}(x)=\operatorname{Cos} x+e^{x}
\end{array}\right.
\end{aligned}
$$

Therefore, we get the result as,

$$
\left\{\begin{array}{l}
y_{1}(x)=e^{x} \\
y_{2}(x)=\sin x, \\
y_{3}(x)=\operatorname{Cos} x+e^{x},
\end{array}\right.
$$

This is the same result obtained by ADM in [22].

Example 4.2 Consider the non-linear system of differential equations,

$$
\begin{aligned}
& \frac{d y_{1}}{d x}=2 y_{2}^{2}, \\
& \frac{d y_{2}}{d x}=e^{-x} y_{1}, \\
& \frac{d y_{3}}{d x}=y_{2}+y_{3},
\end{aligned}
$$

subjected to the initial conditions

$$
y_{1}(0)=1, y_{2}(0)=1, y_{3}(0)=0 \text {. }
$$

The exact solution is given, $y_{1}=e^{2 x}, y_{2}=e^{x}$, and $y_{3}=x e^{x}$. According to VIM, the correction functional for the Eq. (10), (11) \& (12) can be written as 
Jamshad Ahmed and Faizan Hussain/ BIBECHANA 13 (2016) 77-86: RCOST p.81 (Online Publication: Dec., 2015)

$$
\left\{\begin{array}{l}
y_{1, n+1}(x)=y_{1, n}(x)+\int_{0}^{x} \lambda_{1}(x, \tau)\left(\frac{d y_{1, n}(\tau)}{d \tau}-2 \tilde{y}_{2, n}^{2}\right) d \tau, \\
y_{2, n+1}(x)=y_{2, n}(x)+\int_{0}^{x} \lambda_{2}(x, \tau)\left(\frac{d y_{2, n}}{d \tau}-e^{-\tau} \tilde{y}_{1, n}\right) d \tau, \\
y_{3, n+1}(x)=y_{3, n}(x)+\int_{0}^{x} \lambda_{3}(x, \tau)\left(\frac{d y_{3, n}}{d \tau}-\tilde{y}_{2, n}-\tilde{y}_{3, n}\right) d \tau,
\end{array}\right.
$$

The Lagrange multiplier $\lambda_{i}(x, \tau), i=1,2,3$ can be identified via Variational Theory; i.e. the multiplier should be chosen in such a way that the correction functional equation is stationary i.e. $\delta y_{1, n+1}(x)=0, \delta y_{2, n+1}(x)=0 \& \delta y_{3, n+1}(x)=0$ So from Eq. (13), we get

$$
\lambda_{1}(x, \tau)=-1, \lambda_{2}(x, \tau)=-1, \text { and } \lambda_{3}(x, \tau)=-1,
$$

Thus Eq. (13) becomes

$$
\left\{\begin{array}{l}
y_{1, n+1}(x)=y_{1, n}(x)+\int_{0}^{x}(-1)\left(\frac{d y_{1, n}(\tau)}{d \tau}-2\left(y_{2, n}\right)^{2}\right) d \tau, \\
y_{2, n+1}(x)=y_{2, n}(x)+\int_{0}^{x}(-1)\left(\frac{d y_{2, n}}{d \tau}-e^{-\tau} y_{1, n}\right) d \tau, \\
y_{3, n+1}(x)=y_{3, n}(x)+\int_{0}^{x}(-1)\left(\frac{d y_{3, n}}{d \tau}-y_{2, n}-y_{3, n}\right) d \tau,
\end{array}\right.
$$

According to VIMHP, Eq. (14) can be written as

$$
\begin{aligned}
& \left(\sum_{n=0}^{\infty} p^{n} y_{1, n}(x)=y_{1,0}(x)+p \int_{0}^{x}(-1)\left(\sum_{n=0}^{\infty} p^{n} \frac{d y_{1, n}(\tau)}{d \tau}-2 \sum_{n=0}^{\infty} p^{n}\left(y_{2, n}\right)^{2}\right) d \tau,\right. \\
& \left\{\sum_{n=0}^{\infty} p^{n} y_{2, n}(x)=y_{2,0}(x)+p \int_{0}^{x}(-1)\left(\sum_{n=0}^{\infty} p^{n} \frac{d y_{2, n}}{d \tau}-e^{-\tau} \sum_{n=0}^{\infty} p^{n} y_{1, n}\right) d \tau,\right. \\
& \sum_{n=0}^{\infty} p^{n} y_{3, n}(x)=y_{3,0}(x)+p \int_{0}^{x}(-1)\left(\sum_{n=0}^{\infty} p^{n} \frac{d y_{3, n}}{d \tau}-\sum_{n=0}^{\infty} p^{n} y_{2, n}-\sum_{n=0}^{\infty} p^{n} y_{3, n}\right) d \tau, \\
& \left(\sum_{n=0}^{\infty} p^{n} y_{1, n}(x)=1+p \int_{0}^{x}(-1)\left(\sum_{n=0}^{\infty} p^{n} \frac{d y_{1, n}(\tau)}{d \tau}-2 \sum_{n=0}^{\infty} p^{n} y_{2, n}^{2}\right) d \tau,\right. \\
& \left\{\sum_{n=0}^{\infty} p^{n} y_{2, n}(x)=1+p \int_{0}^{x}(-1)\left(\sum_{n=0}^{\infty} p^{n} \frac{d y_{2, n}}{d \tau}-e^{-\tau} \sum_{n=0}^{\infty} p^{n} y_{1, n}\right) d \tau,\right. \\
& \left(\sum_{n=0}^{\infty} p^{n} y_{3, n}(x)=p \int_{0}^{x}(-1)\left(\sum_{n=0}^{\infty} p^{n} \frac{d y_{3, n}}{d \tau}-\sum_{n=0}^{\infty} p^{n} y_{2, n}-\sum_{n=0}^{\infty} p^{n} y_{3, n}\right) d \tau,\right.
\end{aligned}
$$


Now, comparing the co-efficient of like powers of $p$,

$$
\begin{aligned}
& p^{(0)}: \quad\left\{\begin{array}{l}
y_{1,0}(x)=1 \\
y_{2,0}(x)=1, \\
y_{3,0}(x)=0
\end{array}\right. \\
& p^{(1)}:\left\{\begin{array}{l}
y_{1,1}(x)=\int_{0}^{x}(-1)\left(\frac{d y_{1,0}}{d \tau}-2\left(y_{2,0}\right)^{2}\right) d \tau \\
y_{2,1}(x)=\int_{0}^{x}(-1)\left(\frac{d y_{2,0}}{d \tau}-e^{-\tau} y_{1,0}\right) d \tau, \\
y_{3,1}(x)=\int_{0}^{x}(-1)\left(\frac{d y_{3,0}}{d \tau}-y_{2,0}-y_{3,0}\right) d \tau
\end{array}\right. \\
& : \quad\left\{\begin{array}{l}
y_{1,1}(x)=2 x \\
y_{2,1}(x)=1-e^{-x} \\
y_{3,1}(x)=x
\end{array}\right. \\
& p^{(2)}:\left\{\begin{array}{l}
y_{1,2}(x)=-4+4 x+4 e^{-x} \\
y_{2,2}(x)=2-2 e^{-2 x}-2 x e^{-x} \\
y_{3,2}(x)=-1+e^{-x}+x+\frac{x^{2}}{2}
\end{array}\right. \\
& p^{(3)}:\left\{\begin{array}{l}
y_{1,3}(x)=-15+10 x+12 e^{-x}+3 e^{-2 x}+8 x e^{-x} \\
y_{2,3}(x)=2-2 e^{-2 x}-4 x e^{-x} \\
y_{3,3}(x)=-2+2 x e^{-x}+2 x e^{-x}+e^{-2 x}+x+\frac{x^{2}}{2}+\frac{x^{3}}{6}
\end{array},\right.
\end{aligned}
$$

Therefore, approximations to the solutions with the five terms are as follows:

$$
\left\{\begin{array}{l}
y_{1}(x) \approx 4(26 x+31) e^{-x}+\left(-4 x^{2}-6 x+15\right) e^{-2 x}-3.11111 e^{-3 x}+58.6666 x-134.888 \\
y_{2}(x) \approx-4(8 x-7) e^{-x}-8(2 x+5) e^{-2 x}+0.111111(12 x+13) e^{-3 x}+11.5555 \\
y_{3}(x) \approx(12 x+3) e^{-x}+(2 x+7.5) e^{-2 x}-0.111111 e^{-3 x}+0.0083333 x^{5}+x^{2}+4.6666 x-10.3888
\end{array}\right.
$$

This is same as obtain by ADM in [22]. 
Jamshad Ahmed and Faizan Hussain/ BIBECHANA 13 (2016) 77-86: RCOST p.83 (Online Publication: Dec., 2015)

Table1. Numerical values of these solution.

\begin{tabular}{lllllll}
\hline$x_{i}$ & $y_{1}\left(x_{i}\right)$ & $e y_{1}\left(x_{i}\right)$ & $y_{2}\left(x_{i}\right)$ & $e y_{2}\left(x_{i}\right)$ & $y_{3}\left(x_{i}\right)$ & $e y_{3}\left(x_{i}\right)$ \\
\hline 0 & 1.00008 & 0 & 1 & 0 & 0 & 0 \\
0.1 & 1.22132 & $1.6535 \mathrm{E}-5$ & 1.10516 & $2.9323 \mathrm{E}-6$ & 0.110517 & 0 \\
0.2 & 1.49186 & $5.3375 \mathrm{E}-5$ & 1.22139 & $1.1211 \mathrm{E}-5$ & 0.244275 & 0 \\
0.3 & 1.82161 & $5.9740 \mathrm{E}-4$ & 1.34974 & $1.1407 \mathrm{E}-4$ & 0.404906 & $5.1165 \mathrm{E}-5$ \\
0.4 & 2.22249 & $3.1315 \mathrm{E}-3$ & 1.49125 & $5.7298 \mathrm{E}-4$ & 0.594560 & $2.7328 \mathrm{E}-4$ \\
0.5 & 2.70702 & $1.1341 \mathrm{E}-2$ & 1.64676 & $1.9591 \mathrm{E}-3$ & 0.823372 & $9.8853 \mathrm{E}-4$ \\
0.6 & 3.28813 & $3.2068 \mathrm{E}-2$ & 1.81686 & $5.2497 \mathrm{E}-3$ & 1.090460 & $2.8076 \mathrm{E}-3$ \\
0.7 & 3.97860 & $7.6660 \mathrm{E}-2$ & 2.00184 & $1.1909 \mathrm{E}-2$ & 1.402860 & $6.7609 \mathrm{E}-3$ \\
0.8 & 4.79050 & $6.6253 \mathrm{E}-1$ & 2.20161 & $2.3929 \mathrm{E}-2$ & 1.766030 & $1.1440 \mathrm{E}-2$ \\
0.9 & 5.73528 & $3.1445 \mathrm{E}-1$ & 2.41576 & $4.3841 \mathrm{E}-2$ & 2.185680 & $2.7962 \mathrm{E}-2$ \\
\hline
\end{tabular}

\section{Graphical representation:}

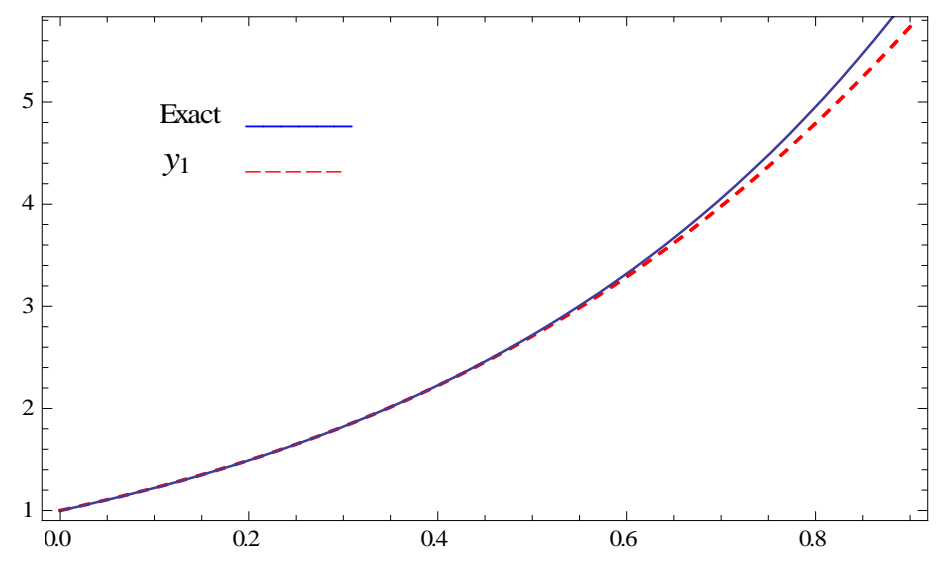

Fig. 1: Comparison of exact and approximate solution of $y_{1}$

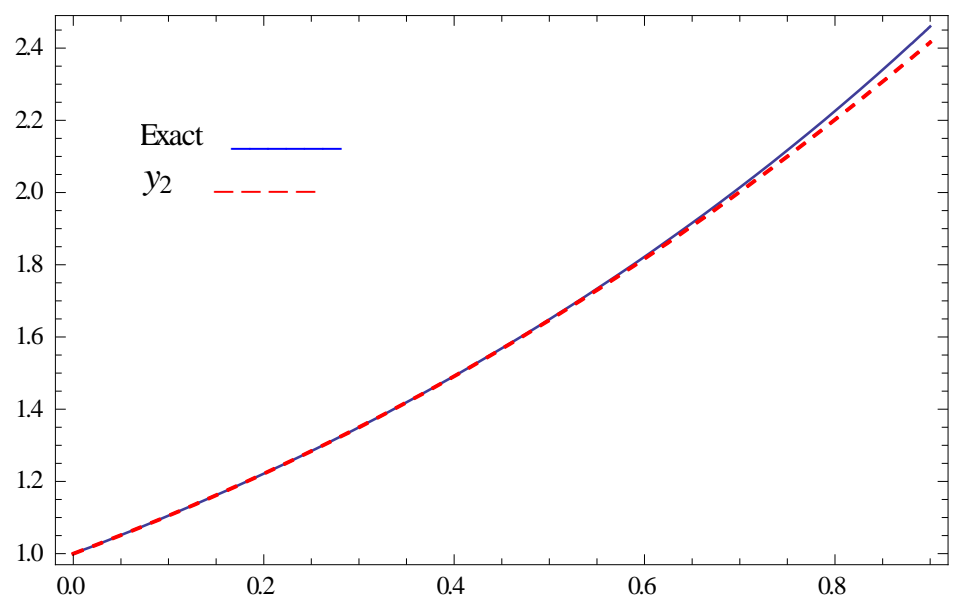

Fig. 2: Comparison of exact and approximate solution of $y_{2}$, 
Jamshad Ahmed and Faizan Hussain/ BIBECHANA 13 (2016) 77-86: RCOST p.84 (Online Publication: Dec., 2015)

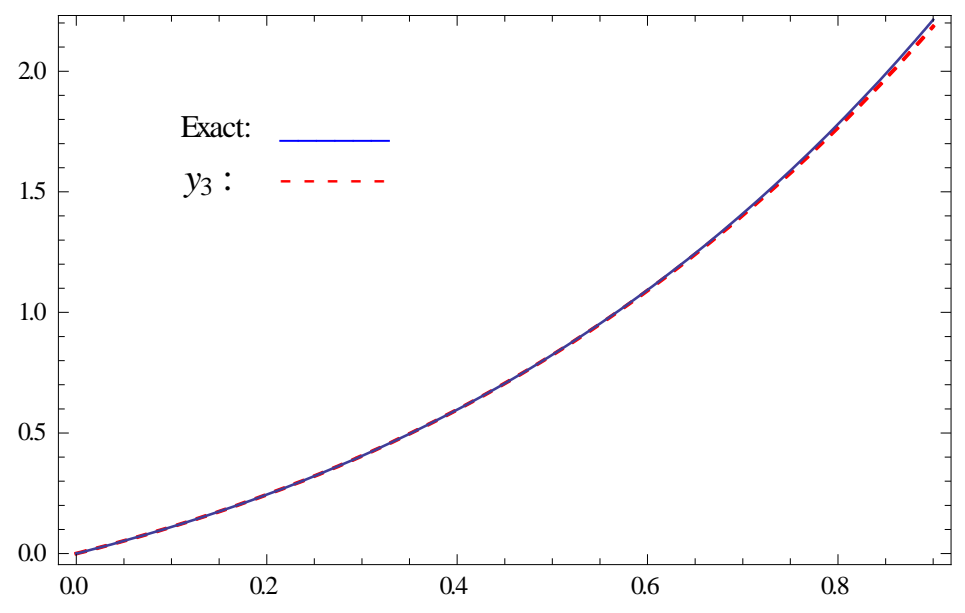

Fig. 3: Comparison of exact and approximate solution of $\mathrm{y}_{3}$.

Example 4.3 Consider a non-linear ordinary differential equation

$$
\frac{d^{3} y}{d x^{3}}=\frac{1}{x} y+\frac{d y}{d x}
$$

subject to the boundary conditions

$$
y(0)=0, y^{\prime}(0)=1, y^{\prime \prime}(0)=2 \text {. }
$$

Considering $y_{1}(x)=y(x), y_{2}(x)=y^{\prime}(x)$, and $y_{3}(x)=y^{\prime \prime}(x)$, we convert Eq. (16) in the system of nonlinear of three differential equation of order one, i.e.

$$
\begin{aligned}
& y_{1}^{\prime}(x)=y_{2}(x), \\
& y_{2}^{\prime}(x)=y_{3}(x), \\
& y_{3}^{\prime}(x)=\frac{1}{x} y_{1}(x)+y_{3}(x),
\end{aligned}
$$

According to VIM, the correction functional for the Eq. (17), (18) \& (19) can be written as,

$$
\left\{\begin{array}{l}
y_{1, n+1}(x)=y_{1, n}(x)+\int_{0}^{x} \lambda(x, \tau)\left(\frac{d y_{1, n}(\tau)}{d \tau}-\tilde{y}_{2, n}\right) d \tau, \\
y_{2, n+1}(x)=y_{2, n}(x)+\int_{0}^{x} \lambda(x, \tau)\left(\frac{d y_{2, n}}{d \tau}-\tilde{y}_{3, n}\right) d \tau, \\
y_{3, n+1}(x)=y_{3, n}(x)+\int_{0}^{x} \lambda(x, \tau)\left(\frac{d y_{3, n}}{d \tau}-\frac{1}{\tau} \tilde{y}_{1, n}-\tilde{y}_{3, n}\right) d \tau,
\end{array}\right.
$$


The Lagrange multiplier $\lambda_{i}(x, \tau), i=1,2,3$ can be identified via variational theory. i.e. the multiplier should be chosen in such a way that the correction functional equation is stationary i.e. $\delta y_{1, n+1}(x)=0, \delta y_{2, n+1}(x)=0 \& \delta y_{3, n+1}(x)=0$ So from Eq. (20), we get

$$
\lambda_{1}(x, \tau)=-1, \lambda_{2}(x, \tau)=-1, \text { and } \lambda_{3}(x, \tau)=-1,
$$

Thus Eq. (20) becomes

$$
\left\{\begin{array}{l}
y_{1, n+1}(x)=y_{1, n}(x)+\int_{0}^{x}(-1)\left(\frac{d y_{1, n}(\tau)}{d \tau}-\tilde{y}_{2, n}\right) d \tau, \\
y_{2, n+1}(x)=y_{2, n}(x)+\int_{0}^{x}(-1)\left(\frac{d y_{2, n}}{d \tau}-\tilde{y}_{3, n}\right) d \tau, \\
y_{3, n+1}(x)=y_{3, n}(x)+\int_{0}^{x}(-1)\left(\frac{d y_{3, n}}{d \tau}-\frac{1}{\tau} \tilde{y}_{1, n}-\tilde{y}_{3, n}\right) d \tau,
\end{array}\right.
$$

Therefore, we get,

$$
\left\{\begin{array}{l}
y_{1,0}(x)=0 \\
y_{2,0}(x)=1 \\
y_{3,0}(x)=2
\end{array}\right.
$$

Let $y^{r}=y_{1,0}+y_{1,1}+y_{1,2}+\cdots+y_{1, r}$ is a notation for an approximation to the solution with $p+1$ term. Therefore, some computed approximations are as follows

$$
\begin{aligned}
& y^{3}=x\left(1+x+\frac{x^{2}}{3}\right), \\
& y^{4}=x\left(1+x+\frac{x^{2}}{2}+\frac{x^{3}}{12}\right), \\
& y^{5}=x\left(1+x+\frac{x^{2}}{2}+\frac{x^{3}}{6}+\frac{x^{4}}{60}\right), \\
& y^{6}=x\left(1+x+\frac{x^{2}}{2}+\frac{x^{3}}{6}+\frac{7 x^{4}}{180}+\frac{x^{5}}{360}\right), \\
& \vdots
\end{aligned}
$$

The closed form solution is

$$
y(x)=x e^{x}
$$

This is the exact solution. 
Jamshad Ahmed and Faizan Hussain/ BIBECHANA 13 (2016) 77-86: RCOST p.86 (Online Publication: Dec., 2015)

\section{Conclusion}

In this paper, Variational Iteration Method using He's polynomials has been implemented successfully to find exact and approximate solutions of linear and nonlinear system of ordinary differential equations. Three numerical examples have been presented to show that this technique is promising. All the calculations are performed easily. Therefore, this method can be applied to many other complicated nonlinear systems of ODEs and PDEs.

\section{References}

[1] J. H. He, International Journal of Modern Physics B, 20 (10) (2006) 1141-1199.

[2] J. H. He, Applied Mathematics and Computation, 114 (2000) 115-123.

[3] S. Momani, S. Abuasad, Chaos, Solitons and Fractals, 27 (2006) 1119-1123.

[4] M.A. Abdou, A.A. Soliman, Journal of Computational and Applied Mathematics, 181 (2005) 245-251.

[5] S. Momani, Z. Odibat, Physics Letters A, 355 (2006) 271-279

[6] M. Dehghan, F. Shakeri, Communications in Numerical Methods in Engineering, (2008) (in press).

[7] A. Saadatmandi, M. Dehghan, Computers and Mathematics with Applications, 58 (11-12) (2009) 21902196.

[8] O. Kiymaz, Int. J. Contemp. Math. Sciences, (5) (37) (2010) 1819 - 1826.

[9] M. Dehghan, A. Saadatmandi, Chaos, Solitons and Fractals, (2008) (in press).

[10] S.A. Yousefi, A. Lotfi, M. Dehghan, Computers and Mathematics with Applications, 58 (11-12) (2009) 2172-2176.

[11] S.A. Yousefi, M. Dehghan, International Journal of Computer Mathematics, (2008) (in press).

[12] A.M. Wazwaz, Central European Journal of Engineering, 4(1) (2014) 64-71.

[13] A. Neamaty and R. Darzi, Boundary Value Problems 2010, 2010: 317369

DOI:10.1155/2010/317369

[14] H. Ozer, International Journal of Nonlinear Sciences and Numerical Simulation, 8 (2007) 513-518.

[15] J. Biazar, H. Ghazvini, International Journal of Nonlinear Sciences and Numerical Simulation, 8 (2007) 311314.

[16] Z.M. Odibat, S. Momani, International Journal of Nonlinear Sciences and Numerical Simulation, 7 (2007) 27-34.

[17] J. Ahmad, and S. T. Mohyud-Din, Bibechana, (12) (2015) 59-69,

[18] M. Dehghan, F. Shakeri, New Astronomy, 13 (2008) 53-59.

[19] G.Y Wang, J. H. He' L.F. Mo, LETTER, Lat. Am. J. Solids Struct., 11 (2) (2014).

[20] J. Ahmad, I. Ahmad and B. Ahmad, 21 ( 2013) 1-15.

[21] S. T. Mohyud-Din and M. A. Noor, J. Appl. Math. Comp., (2008)

DOI: $10.1007 / \mathrm{s} 12190-008-0212-7$

[22] J. Biazar, E. Babolian, R. Islam, Appl. Math. Comp., 147 (2004) 713-719. 